\title{
L'éternel casse-tête
}

\author{
Valérie Junod ${ }^{\text {a }}$, Carole-Anne Baud ${ }^{b}$ \\ a Professeure de droit aux Universités de Genève et de Lausanne; ${ }^{b}$ Doctorante à la Faculté de droit de Genève
}

Le 9 mai dernier, le remboursement par l'assurance de base d'un médicament non inclus dans la liste des médicaments remboursés (LS) ${ }^{1}$ a donné lieu à un nouvel arrêt du Tribunal fédéral ${ }^{2}$. L'affaire opposait une caissemaladie à une patiente souffrant d'une maladie orpheline, la protoporphyrie érythropoïétique. Les personnes atteintes de cette maladie génétique ne supportent l'exposition ni au soleil, ni à certaines lumières artificielles.

La patiente demandait le remboursement du Scenesse (DCI afamelanotide), un médicament autorisé dans l'Union européenne $e^{3}$, mais pas en Suisse 4 . Or les médicaments non autorisés en Suisse ne peuvent pas être inscrits sur la LS et ne sont dès lors en principe pas à charge de l'assurance-maladie obligatoire (AOS). Des exceptions sont toutefois possibles aux conditions fixées par l'art. 71c (art. 71b al. 2 à l'époque des faits) de l'Ordonnance sur l'assurance-maladie (OAMal) ${ }^{5}$. Cette disposition ne distingue pas entre médicaments orphelins et médicaments ordinaires. Invoquant cette ordonnance, la patiente réclamait le remboursement de cinq traitements de Scenesse par année au prix requis par le fabricant, soit 18989 CHF par traitement. La caisse, quant à elle, avait accepté de payer 6560 CHF par traitement au maximum quatre fois par année, laissant la patiente payer la différence. Le tribunal cantonal avait «coupé la poire en

\section{Eine unendliche Geschichte}

Das Bundesgericht wies im Mai dieses Jahres den Fall einer Patientin, die unter erythropoetischer Protoporphyrie, einer seltenen und schmerzhaften Lichtempfindlichkeit, leidet, an die kantonale Vorinstanz zurück. Die Patientin verlangte die vollständige Vergütung der Behandlungskosten von gegen 100000 CHF pro Jahr. Das Bundesgericht fällte keinen Entscheid über die Aufteilung der Kosten, sondern verlangte weitere Abklärungen über den Nutzen des Medikaments. Der entsprechende Nachweis ist jedoch gerade bei seltenen Erkrankungen schwer zu erbringen. Die Politik muss sich nun der Frage stellen, wo die Nutzenschwelle für die Vergütung teurer Behandlungen anzusetzen sei. Hilfreich wäre zum Beispiel die Gründung einer Agentur, die transparente und partizipative Prozesse zur Nutzenbewertung in die Wege leitet und dabei eng mit anderen europäischen Ländern zusammenarbeitet. deux», en retenant que quatre traitements devaient être payés au prix plein de 18989 CHF chacun. Les deux parties ayant fait recours, le Tribunal fédéral a été appelé à trancher.

Malheureusement, le Tribunal fédéral n'a pas - ou finalement peu - tranché. Pour l'essentiel, il renvoie l'affaire au tribunal inférieur cantonal pour que celui-ci réunisse les faits nécessaires à une meilleure compréhension de l'état de santé de l'assurée (i.e., sa situation avec et sans traitement). Parce qu'à maints égards l'arrêt laisse le lecteur sur sa faim, il appelle des commentaires sur trois aspects.

\section{Quelle autorisation étrangère pour être remboursé en Suisse?}

Premièrement, le Tribunal se prononce sur le type d'autorisation étrangère admissible pour une prise en charge exceptionnelle à teneur de l'art. 71b al. 2 OAMal. Cet article exige qu'un médicament importé soit auto-

Le système atteint ses limites si chaque société pharmaceutique innovante entend réclamer un prix de monopole.

risé dans un pays ayant un système d'autorisation de mise sur le marché (AMM) équivalent au système suisse $^{6}$. La disposition ne précise pas le type d'AMM requis. Dans le cas du Scenesse, le médicament n'avait pas reçu pour l'Union européenne une AMM ordinaire, mais une autorisation dite "sous conditions exceptionnelles». Cette procédure est mise en œuvre lorsque le fabricant démontre qu'il ne peut objectivement pas «fournir des renseignements complets sur l'efficacité et la sécurité dans des conditions normales d'emploi», notamment au motif que la maladie étudiée est très rare $^{7}$. Les conditions pour obtenir l'autorisation exceptionnelle sont donc «allégées» par rapport à l'AMM standard. En l'espèce, les essais cliniques portant sur le Scenesse avaient été conduits sur une période courte et des réserves avaient été exprimées sur différents points méthodologiques. Aussi, l'AMM accordée par l'EMA (European Medicines Agency) avait été assortie de l'exigence de mener des études supplémentaires et soumise à réévaluation annuelle. Dans son arrêt, le 


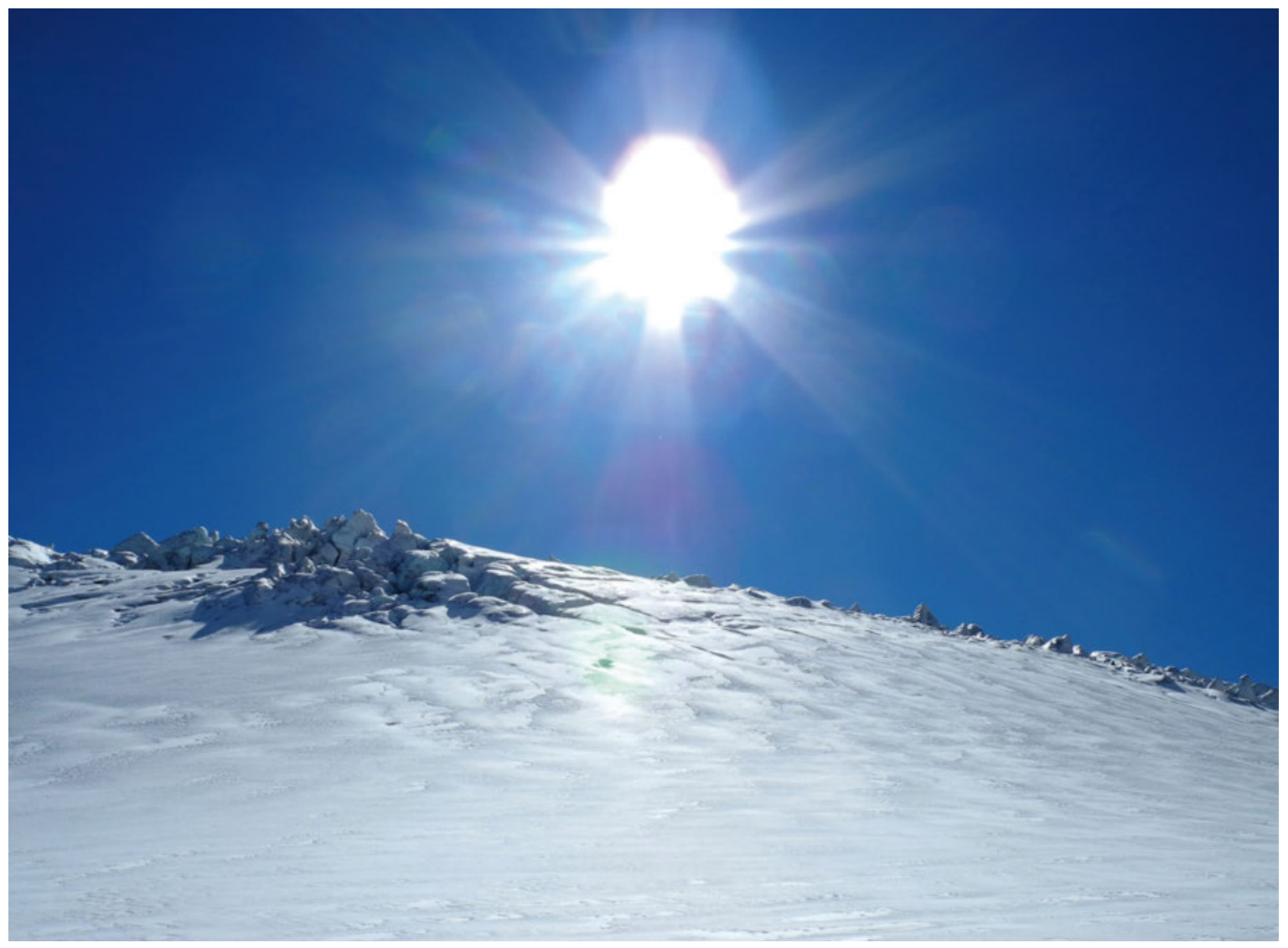

La lumière est insupportable pour les patients souffrant de la protoporphyrie érythropoïétique.

Tribunal fédéral retient que la procédure moins stricte appliquée par l'EMA satisfait tout de même l'exigence de l'art. 71b al. 2 OAMal $^{8}$.

Le raisonnement du Tribunal fédéral est malheureusement succinct; l'importance de l'enjeu aurait appelé des développements plus amples. Un médicament autorisé au terme d'une procédure simplifiée mérite-t-il d'être

\section{On aurait aimé saisir où se situe le seuil du bénéfice thérapeutique élevé.}

remboursé au même titre qu'un médicament pour lequel les preuves d'efficacité sont avérées? A notre avis, s'agissant à tout le moins $d u$ principe de la prise en charge (par opposition à celui du montant à rembourser), la réponse doit être positive. Lorsque l'EMA accorde une autorisation sous conditions exceptionnelles, elle retient que le rapport bénéficerisque est globalement favorable, même si ce constat repose sur des preuves moins fiables que dans les cas ordinaires. Or, le but de l'exigence d'une AMM dans un pays appliquant un système équivalent (art. 71b al. 2 OAMal) est de garantir aux patients un niveau de qualité, de sécurité et d'efficacité équivalant à celui dont ils bénéficient en Suisse. L'évaluation - certes non-définitive-de l'EMA fournit une garantie suffisante.

\section{Quel bénéfice thérapeutique pour être remboursé en Suisse?}

Le Tribunal fédéral aborde ensuite la question du caractère élevé du bénéfice thérapeutique du médicament, seconde exigence fixée par l'OAMal ${ }^{9}$. Ce bénéfice élevé doit être démontré à la fois dans l'abstrait, c'est-àdire sur la base d'essais cliniques, et dans le cas particulier de la patiente assurée ${ }^{10}$.

Quelle que soit la réponse à ces questions, l'enjeu de la répartition du budget décidé demeure.

Le Tribunal admet le bénéfice thérapeutique élevé du Scenesse dans l'abstrait, mais sa motivation tient simplement à ce que le médicament est autorisé dans l'UE en tant que médicament orphelin et que, pour cette catégorie, les exigences d'efficacité nécessaires pour conclure à un bénéfice thérapeutique élevé ne sont pas aussi strictes que pour les autres médicaments ${ }^{11}$. 
Encore une fois regrettablement, le Tribunal fédéral n'explique pas quelle preuve d'efficacité doit être jugée suffisante. Dans le cas du Scenesse, un essai clinique ayant recruté 93 patients avait mis en évidence une tolérance (sans douleur) à la lumière directe passant de 61 heures à 116 heures sur une période de six mois, donc au total moins de deux heures d'exposition par jour pour les patients sous traitement ${ }^{12}$. Par comparaison, dans l'arrêt dit Myozyme de 2010, le Tribunal fédéral avait considéré qu'augmenter la distance de marche de quelques 28 mètres (ou 8,4\%) pour des patients souffrant de la maladie (orpheline) de Pompe ne constituait pas un bénéfice thérapeutique élevé ${ }^{13}$. Les conclusions du Tribunal diffèrent dans ces deux affaires et l'on au-

Dans l'intervalle, et comme solution de substitution temporaire, le législateur suisse devrait définir un seuil.

rait aimé saisir où se situe le seuil du bénéfice thérapeutique élevé. Tant que le Tribunal n'aura pas cerné les contours de cette notion, les caisses-maladie conserveront une grande liberté dans leur interprétation du bénéfice thérapeutique élevé, ce qui nuit à la sécurité juridique et à l'égalité de traitement entre les assurés. S'agissant du bénéfice thérapeutique dans le cas particulier de la patiente, le Tribunal estime nécessaire de connaître en détail l'état de santé de cette dernière avant le début de la thérapie, pendant celle-ci, et une fois celle-ci achevée; pour ce faire, il renvoie la cause au tribunal inférieur tessinois chargé de recueillir ces faits ${ }^{14}$. Il ajoute que l'avis du médecin traitant de la patiente est à considérer avec prudence, car ce dernier a tendance à prendre parti pour ses patients. Mais si le médecin traitant du patient ne peut pas se charger de l'évaluation requise, qui doit évaluer le bénéfice thérapeutique dans le cas particulier? Le médecin-conseil? Et comment cette évaluation peut-elle être menée rétroactivement?

Vu le nombre de cas soumis aux caisses (entre 6000 et 8000 par an $)^{15}$, il serait utile que le législateur ou le Tribunal fédéral dégage les critères pertinents pour conclure à un bénéfice thérapeutique élevé. Faut-il utiliser le critère des années de vie gagnées en bonne santé grâce au traitement? Faut-il tenir compte de la souffrance déjà endurée par le patient sans traitement? De son âge (une maladie qui frappe un enfant doit-elle être appréciée de la même manière que celle qui touche une personne âgée)? De son espérance de vie résiduelle (faut-il tout faire pour celui qui n'a que quelques mois ou années à vivre)? De la rareté de la maladie? De son impact sur la vie sociale du patient, voire sur celle de son entourage? Ces interrogations éthiques sont fon- damentales pour toute société et il n'est pas normal que la mission d'y répondre soit déléguée aux caissesmaladie.

\section{Quel prix pour être remboursé en Suisse?}

Troisième aspect à souligner du jugement: le prix à payer. Le Tribunal fédéral élude entièrement cet enjeu du coût. Il profite de ce que les faits doivent de toute façon être davantage élucidés par le tribunal cantonal pour ne pas aborder ce thème. Pourtant, c'était le point principal de désaccord entre les parties, la caisse estimant ne devoir payer qu'un tiers du coût exigé par le fabricant.

Sur le coût à assumer par l'AOS pour un médicament hors LS, jusqu'en mars 2017, l'OAMal était remarquablement peu diserte - à tel point qu'on pouvait soupçonner une attitude délibérée du Conseil fédéral. En effet, l'art. 71b OAMal dans sa formulation valable à l'époque de l'arrêt exigeait un rapport coût-bénéfice "adéquat». Mais lorsqu'il s'agit de décider si la caisse doit payer 6560 CHF, 18989 CHF ou encore une somme différente, le terme «adéquat» n'est guère utile.

\section{Quelle solution?}

Une affaire comme celle-ci jette une lumière crue sur le phénomène aujourd'hui inéluctable de la gestion des coûts et du rationnement des soins dans l'assurancemaladie. Certes, dans un monde idéal, chaque patient obtiendrait toutes les prestations médicales (mais aussi sociales) qui lui permettent d'optimiser sa santé, comprise comme un état complet de bien-être ${ }^{16}$. Cependant, un tel résultat impliquerait des primes d'assurance et/ou une charge fiscale que peu d'assurés ou de contribuables seraient d'accord d'assumer. Les dépenses de santé représentent aujourd'hui 11\% du PIB en Suisse $^{17}$. Doit-on considérer ce taux comme adéquat? Au contraire, trop élevé? Trop bas? Doit-on augmenter l'enveloppe totale pour permettre notamment aux patients atteints de maladies rares d'accéder à des thérapies de plus en plus chères?

Quelle que soit la réponse à ces questions, l'enjeu de la répartition du budget décidé demeure. Faut-il mettre l'accent sur le remboursement des médicaments utilisés par le plus grand nombre même s'ils ne sauvent que rarement des vies? Faut-il au contraire donner la priorité au petit nombre de patients les plus durement atteints dans leur santé? Formulé différemment, faut-il sortir la «bobologie» (encore un terme sans définition...) des prestations de l'assurance-maladie pour concentrer les ressources sur les maladies les plus sérieuses? 
Une chose est sûre, ces questions ultra-sensibles ne pourront pas être esquivées encore longtemps. Les nouveaux médicaments qui entrent sur le marché affichent des prix de plus en plus élevés. Le cas des nouveaux médicaments contre l'hépatite $\mathrm{C}$ a eu un important retentissement en Suisse comme à l'étranger, en raison de leur prix de départ d'environ 80000 CHF par traitement. Parmi les nouveaux médicaments aux prix très élevés, on peut citer entre autres le Keytruda à environ 110000 CHF par an par patient («pa/pp») ${ }^{18}$ ou les médicaments traitant des maladies rares comme le Myozyme (env. $370000 \mathrm{CHF} \mathrm{pa} / \mathrm{pp}^{19}$ ), le Soliris (env. 360000 CHF pa/pp ${ }^{20}$ ), le Kalydeco (env. 295000 CHF $\mathrm{pa} / \mathrm{pp}^{21}$ ) ou le Kyprolis (env. 100000 CHF pa/pp ${ }^{22}$ ). Même des anciens médicaments voient leur prix augmenter, à l'occasion d'une extension d'indication ou d'une reformulation, comme par exemple les solutions injectables de fer ${ }^{23}$.

Chaque entreprise pharmaceutique qui réclame un prix élevé se défend en invoquant tant son investissement coûteux dans la recherche qui a permis de découvrir ledit médicament que la nécessité pour elle de financer ses prochaines innovations ${ }^{24}$. Le système atteint toutefois ses limites si chaque société pharmaceutique innovante entend réclamer un prix de monopole dans un contexte où la demande est totalement inélastique et rendue solvable par l'assurance-sociale. Pour faire face à ces défis, un débat social et politique doit être lancé. Nous estimons préférable qu'un outil, même imparfait (ce qui est au demeurant inévitable), soit défini par le législateur, agréé par le peuple (au besoin via référendum), explicité par les tribunaux (sur recours), pour être enfin mis en œuvre de manière transparente par une agence publique. L'alternative qui consiste à laisser chaque caisse décider - au cas par cas et avec une marge de manœuvre considérable - les requêtes qui lui sont soumises n'est pas éthiquement acceptable.

\section{Une approche transparente et participative}

sous www.bullmed.ch $\rightarrow$ Numéro actuel ou

$\rightarrow$ Achives $\rightarrow 2017$

$\rightarrow$ Numéro 2017/36.

Correspondance:

Prof. Dr Valérie Junod

Université de Lausanne-

HEC Anthropole

bureau 3076

CH-1015 Lausanne

valerie.junod[at]unil.ch revanche, l'agence aurait le pouvoir de contraindre la société pharmaceutique qui entend mettre sur le marché un nouveau médicament à soumettre les pièces et données dont elle dispose ${ }^{25}$. En particulier, elle aurait le pouvoir d'exiger des comptes audités sur ce que l'entreprise a dépensé pour développer ledit médicament ${ }^{26}$. Une telle approche devrait être transparente et participative, à l'instar de ce que fait par exemple le National Institute for Health and Care Excellence (NICE) anglais, l'Institut für Qualität und Wirtschaftlichkeit im Gesundheitswesen (IQWiG) allemand ou l'Institute for Clinical and Economic Review (ICER) américain. Le travail de l'agence devrait être mené en étroite collaboration avec les agences qui délivrent l'AMM et si possible en même temps ou immédiatement après.

Nous sommes évidemment conscientes qu'une telle proposition nécessitera des années pour être discutée, puis éventuellement mise en place. Dans l'intervalle, et comme solution de substitution temporaire, le législateur suisse devrait définir un seuil - ne serait-ce que le très décrié $100000 \mathrm{CHF}$ par $\mathrm{QALY}^{27}$ - et une méthode d'évaluation - ne serait-ce que la prise en compte d'évaluations étrangères - pour que les milliers de demandes de remboursement de médicaments hors LS puissent être évaluées par les caisses, puis le cas échéant tranchées par les tribunaux, dans le respect de l'égalité de traitement.

\section{Conclusion}

Nous conclurons sur une note qu'on qualifiera de plutôt positive pour les patients atteints de maladies rares. La révision de l'OAMal entrée en vigueur en mars 2017 (mais inapplicable au cas d'espèce ${ }^{28}$ ) permet désormais à l'OFSP d'obtenir des caisses des données sur la manière dont elles traitent les demandes de remboursement des médicaments hors $\mathrm{LS}^{29}$. A terme, la surveillance des pratiques des caisses devrait se trouver renforcée. Par ailleurs, la révision prévoit que si la caisse ou le tribunal conclut que le médicament hors LS doit être remboursé, c'est alors l'entier de son coût effectif qui doit être payé ${ }^{30}$. A cet égard, le nouveau régime introduit le "tout ou rien»: du moment que le rapport coût-bénéfice est approprié, la caisse n'a plus la possibilité de "couper la poire en deux" et de laisser une partie de la charge financière reposer sur le patient. Reste à espérer que cette révision ne conduira pas les caisses à davantage de sévérité.

Crédit photo

(c) Anna Sax 


\section{Notes de bas de page}

1 La liste des médicaments remboursés est appelée liste des spécialités (LS).

2 Arrêt du Tribunal fédéral 9C_711/2016 du 9 mai 2017 (rendu en italien). Le thème du remboursement des médicaments hors LS a déjà été traité notamment dans les arrêts du Tribunal fédéral 8C_523/2016 du 27 octobre 2016 (Synagis), ATF 142 V 325 (Sumatrip$\operatorname{tan),~ATF~} 139$ V 375 (Soliris), ATF 136 V 395 (Myozyme), arrêt du Tribunal fédéral 9C 785/2011 du 25 avril 2012 (Concerta), arrêt du Tribunal fédéral 9C_550/2011 du 23 mars 2012 (Remicade), ATF $130 \mathrm{~V}$ 532 (Herceptin).

3 Le 22 décembre 2014, l'Union européenne a délivré à l'entreprise Clinuvel l'autorisation de mise sur le marché pour le Scenesse.

4 La patiente avait dans un premier temps obtenu l'accès au médicament dans le cadre d'un programme compassionnel, vraisemblablement lancé par la société pharmaceutique, mais celui-ci avait pris fin.

5 RS 832.102. Le $1^{\text {er }}$ mars 2017, une version révisée de l'OAMal est entrée en vigueur. Sur les difficultés que rencontrent les patients souffrant de maladies orphelines à obtenir une prise en charge adéquate de leur santé, voir le très complet article de Franziska Sprecher, Seltene Krankheiten, Eine Herausforderung für das schweizerische Gesundsheitsrecht, Jusletter 19 mai 2014

6 Cf. Swissmedic, Liste de tous les pays ayant institué un système de contrôle des médicaments équivalent, version de juillet 2017. L'importation du médicament par le patient ou son médecin doit par ailleurs respecter les conditions de l'art. 20 al. 2 de la Loi du 15 décembre 2000 sur les produits thérapeutiques (LPTh; RS 812.21) et de l'art. 36 de Ordonnance du 17 octobre 2001 sur les autorisations dans le domaine des médicaments (OAMéd; 812.212.1).

7 Art. 14 al. 8 du Règlement $N^{\circ} 726 / 2004$ du Parlement européen et du Conseil du 31 mars 2004 établissant des procédures communautaires pour l'autorisation et la surveillance en ce qui concerne les médicaments à usage humain et à usage vétérinaire, et instituant une Agence européenne des médicaments; art. 22 de la Directive 2001/83/CE du Parlement européen et du Conseil du 6 novembre 2001 instituant un code communautaire relatif aux médicaments à usage humain.

8 Consid. 9.

9 Art. 71b al. 2 OAMal, par renvoi à l'art. 71 al. 1 let. b OAMal.

10 ATF 136 V 395, consid. 6.4 et 6.5 .

11 Consid.11.3.1.

12 EMA, Summary of the risk management plan (RMP) for Scenesse (afamelanotide), octobre 2014.

13 ATF 136 V 395, consid. 6.7

14 Le tribunal cantonal devra mettre en œuvre une expertise médicale pour déterminer l'intensité des symptômes chez la patiente, son taux de tolérance à la lumière avec et sans le médicament, les activités que l'administration du médicament lui permet de mener et les activités auxquelles elle doit renoncer sans traitement. Cf. consid. 11.3.3 et 11.4.

15 Ces chiffres correspondent à l'estimation haute et basse du nom bre de demandes, entre mars 2011 et juin 2013, pour lesquelles la question du bénéfice thérapeutique élevé s'est posée (demandes fondées sur les art. 71a et 71b OAMal de l'époque). Ils ont été obtenus par un sondage auprès des caisses-maladie. Cf. Rüefli Christian/Bolliger Christian, Off-Label-Use in der obligatorischen Krankenpflegeversicherung - Evaluation der Umsetzung von Artikel 71a und 71b KVV, Bern: Bundesamt für Gesundheit, 2014, p. 13.

16 Sur les bases légales internationales et suisses du droit à la santé, voir notamment la contribution précitée de F. Sprecher.

17 Sur les dépenses pour les médicaments en Suisse et dans neuf autres pays développés, voir la récente analyse de Steven G. Morgan, Christine Leopold et Anita K. Wagner, Drivers of expenditure on primary care prescription drugs in 10 high-income countries with universal health coverage, CMAJ 189(23) p. e794, 2017.
18 Keytruda est un anticancéreux de MSD Merck Sharp \& Dohme. L'estimation du prix pa/pp a été réalisée à partir du prix inscrit dans la LS (1994.25 CHF le flacon de $50 \mathrm{mg}$ ), selon la posologie du compendium. Ce chiffre est confirmé par l'organisation Médecins du monde sur son site https://medecinsdumonde.ch/news/post_ typenewsp6937/.

19 Myozyme (maladie de Pompe/Sanofi-Aventis); prix LS: $587.75 \mathrm{CHF} /$ flacon de $50 \mathrm{mg}$; prix annuel estimé pour un patient de 60 kilos.

20 Soliris (hémoglobinurie paroxystique nocturne/Alexion); prix LS: $5948.80 \mathrm{CHF} /$ flacon de $30 \mathrm{ml}$; prix annuel: cf. Helsana Arzneimittel Report, Ausgabe 2016, p. 56.

21 Kalydeco (mucoviscidose/Vertex Pharmaceuticals); prix LS $22796 \mathrm{CHF} / 56$ pièces; prix annuel estimé pour un patient de plus de $25 \mathrm{~kg}$.

22 Kyprolis (myélome multiple/Amgen); prix LS: 1540.65 CHF/flacon de $60 \mathrm{mg}$; prix annuel estimé pour le dosage maximal.

23 Helsana Arzneimittel Report, Ausgabe 2016, p. 47.

24 Voir pour un aperçu des positions opposées: Samia Hurst, Médicaments: un prix juste? Bull méd suisses. 2016;97(40):1400. Sur la difficulté juridique à fixer le seuil d'un prix inéquitable, voir aussi Adrian Raass, Unangemessene Preise - Zu Theorie und Praxis einer problematischen Bestimmung, Jusletter 22 mai 2017.

25 Des projets de loi ont été lancés aux Etats-Unis afin de contrer ce que les pouvoirs publics estiment être des prix excessifs; voir notamment Jeremy A. Greene et William V. Padula, Targeting unconscionable prescription-drug prices - Maryland's anti-price gouging law, 377(2) New England Journal of Medicine p. 101-3. (2017).

26 Une approche analogue a été mise en œuvre tout récemment par l'Etat du Nevada: sa loi adoptée en juillet dernier permet d'exiger des sociétés pharmaceutiques vendant des médicaments contre le diabète des informations concernant notamment leur coût de fabrication du médicament, leur coût d'acquisition du médicament, leur rabais consenti, leur coût de marketing et publicité leur profit réalisé grâce au médicament. Cf. Nevada Senate Bill No. 539.

27 Le QALY pour «quality-adjusted life year» permet d'apprécier le gain d'années de vie en le pondérant par la qualité de ces années supplémentaires. Un médicament qui permet une année de vie supplémentaire en bonne santé obtient un QALY de 1 , tandis que si cette année supplémentaire est vécue dans un état de santé exécrable, le QALY sera proche de O. Un seuil de 100000 CHF par QALY signifie donc une disposition à payer 100000 CHF pour obtenir une année supplémentaire de vie en bonne santé.

28 A l'époque où la caisse avait dû trancher la demande de son assurée, la nouvelle disposition légale n'était pas en vigueur.

29 Art. 28 al. 3bis OAMal. Les caisses doivent notamment fournir à l'OFSP des données sur le médicament, sur la décision de prise en charge et sur le délai pour traiter la demande. Ces données seront utilisées par l'OFSP pour identifier les médicaments faisant le plus souvent l'objet de demandes de remboursement exceptionnel, pour déterminer le temps pris par les caisses pour rendre leurs décisions et pour estimer si ces décisions sont prises de manière uniforme entre les différentes caisses. Sur la base de ces données, une nouvelle évaluation des art. 71a et suivants OAMal sera effectuée en 2019. Cf. OFSP, OAMal et OPAS, Modifications prévues pour le $1^{\text {er }}$ mars 2017, Commentaire et teneur des modifications, Berne, $1^{\text {er }}$ février 2017, p. 9.

30 Art. 71c al. 2 et art. 71d al. 4 OAMal. 